\title{
Restoring airway epithelial barrier dysfunction: a new therapeutic challenge in allergic airway disease*
}

\author{
Brecht Steelant ${ }^{1}$, Sven F. Seys ${ }^{1}$, Guy Boeckxstaens², Cezmi A. Akdis³, Jan L. \\ Ceuppens ${ }^{1}$, Peter W. Hellings ${ }^{1,4,5,6}$ \\ Rhinology 54: 195-205, 2016 \\ DOI:10.4193/Rhino15.376 \\ *Received for publication: \\ December 20, 2015 \\ Accepted: April 18, 2016 \\ ${ }^{2}$ Translational Research in Gastro Intestinal Disorders, KU Leuven, Leuven, Belgium \\ ${ }^{3}$ Swiss Institute of Allergy and Asthma Research (SIAF), University of Zurich, Davos, Switzerland

\begin{abstract}
An intact functional mucosal barrier is considered to be crucial for the maintenance of airway homeostasis as it protects the host immune system from exposure to allergens and noxious environmental triggers. Recent data provided evidence for the contribution of barrier dysfunction to the development of inflammatory diseases in the airways, skin and gut. A defective barrier has been documented in chronic rhinosinusitis, allergic rhinitis, asthma, atopic dermatitis and inflammatory bowel diseases. However, it remains to be elucidated to what extent primary (genetic) versus secondary (inflammatory) mechanisms drive barrier dysfunction. The precise pathogenesis of barrier dysfunction in patients with chronic mucosal inflammation and its'implications on tissue inflammation and systemic absorption of exogenous particles are only partly understood. Since epithelial barrier defects are linked with chronicity and severity of airway inflammation, restoring the barrier integrity may become a useful approach in the treatment of allergic diseases.
\end{abstract}

We here provide a state-of-the-art review on epithelial barrier dysfunction in upper and lower airways as well as in the intestine and the skin and on how barrier dysfunction can be restored from a therapeutic perspective.

Key words: tight junctions, allergic rhinitis, chronic rhinosinusitis, trans-epithelial electric resistance, fluticasone, EGF signaling

\section{Key points}

- Restoring nasal epithelial barrier function

- Epithelial barrier dysfunction is linked to allergic diseases

- Corticosteroids upregulate tight junction

\section{Introduction}

The airway epithelium is the first site of contact with inhaled particles like allergens or microorganisms. It is a pseudo-stratified columnar epithelial barrier that prevents the penetration of possible harmful particles ${ }^{(1)}$. Besides protecting the host by a physical barrier, airway epithelial cells are an integral part of the innate immune response ${ }^{(2,3)}$. Mucociliary clearance, release of antimicrobial peptides and the production of chemokines and cytokines all contribute to eradicating possible harmful organisms from entering the body by inducing an appropriate immunological response ${ }^{(4)}$. Epithelial cells express different receptors such as toll like receptors (TLR) ${ }^{(5)}$, protease activated receptors, NOD-like receptors and c-type lectin receptors ${ }^{(4)}$. These pattern recognition receptors sense the environment for the presence of danger associated-and pathogen associated molecular patterns and, thereby contribute to the induction of an immune response. The immunological role of airway epithe-

List of Abbreviations. EGF: epidermal growth factor; ERK: extracellular signal-regulated kinases; JAM-A: junctional adhesion molecule A; MAPK:

Mitogen-activated protein kinase; TEER: trans-epithelial electrical resistance; TJ: tight junction; TLR: toll like receptor; TNF: tumor necrosis factor; TSLP: Thymic stromal lymphopoietin; ZO: zonula occludens 


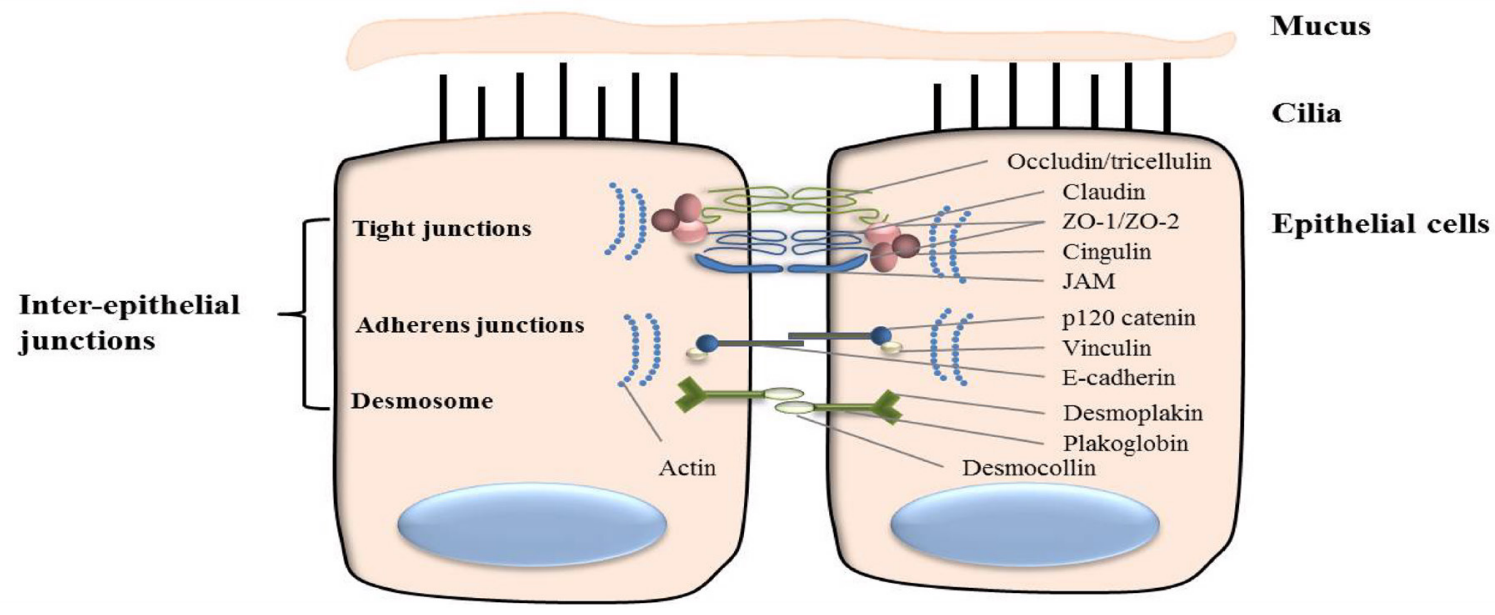

Figure 1. Schematic representation of the different inter-epithelial junctions.

lial cells has nicely been discussed elsewhere ${ }^{(2-4,6)}$.

The physical barrier to the external environment is created by means of different junctional complexes that connect epithelial cells to one another ${ }^{(7,8)}$ (Figure 1). Tight junctions (TJs) are the most apically located epithelial junctions and are key regulators in the homeostasis of ions, water and certain macromolecules ${ }^{(8)}$. TJs form a rate-limiting barrier to inhaled pathogens. Below the TJs are the adherens junctions. Adherens junctions are essential for gene regulation, linked with cell proliferation and differentiation ${ }^{(9)}$ and are important in maintaining cell-cell contact ${ }^{(10)}$. Desmosomes are in close connectivity with adherens junctions and are associated with cell adhesion ${ }^{(11,12)}$, proliferation and differentiation ${ }^{(13)}$.

TJs consist of different transmembrane and intracellular proteins $(7,14,15)$. Transmembrane proteins such as the claudin family ${ }^{(16)}$, junctional adhesion molecule $A$, occludin and tricellulin form heterodimer/homodimers to seal off the paracellular space between airway epithelial cells. In addition, different intracellular scaffold proteins like the zonula occludens (ZO) family, multi-PDZ domain protein 1, membrane-associated guanylate kinase and cingulin connect the transmembrane proteins to the cytoskeleton ${ }^{(8,12)}$. Claudins are four-transmembrane spanning proteins, specialized in the regulation of permeability and $\mathrm{TJ}$ resistance ${ }^{(16)}$. Nowadays, more than 24 claudin members have been identified ${ }^{(16,17)}$. Based on their effect on airway epithelial barrier function, claudins can be divided in two groups: the pore forming claudins, and the barrier forming claudins. The pore forming claudins, such as claudin-2 ${ }^{(18)}$ and claudin-7 ${ }^{(19)}$ increase the permeability to anions/cations, while the barrier forming claudins such as claudin-1 ${ }^{(20)}$ and claudin-4 ${ }^{(21)}$ tighten the epithelial barrier function, with the reduction in cation/anion permeability ${ }^{(22)}$. On top, TJs are linked with two different pathways: the pore pathway and the leak pathway ${ }^{\left({ }^{8}\right)}$. The leak pathway is a low capacity, paracellular route that does not discriminate between solutes on the basis of charge and allows limited flux of large molecules. ZO-1 and occludin have been implicated in leak pathway regulation ${ }^{(8)}$. The pore pathway is a high-capacity, size and charge-selective paracellular route, regulated by the claudin family ${ }^{\left({ }^{8}\right)}$. Taken together, TJs are not only passive structures sealing off the paracellular space between neighboring epithelial cells, but are actively involved in different pathways with distinct functions.

Adherens junctions are composed of extracellular E-cadherin and the cytoplasmic p120-catenin and $\beta$-catenin ${ }^{(7,23)}$. Together with TJ, adherens junctions form an apical junctional complex, essential in controlling epithelial cell-to-cell contact as well as regulation of the actin cytoskeleton, intracellular signaling pathways and transcriptional regulation.

Beneath the apical junctional complex are the desmosomes. Desmosomes are built of desmoglein and desmocollin, which are connected to the cytoskeleton via desmoplakin, plakoglobin and plakophillin ${ }^{(11,13)}$.

A disturbed composition of interepithelial junctions will increase the accessibility of foreign particles to the submucosal region. Impaired airway epithelial barrier function may be an important, yet underrated, key player in the pathogenesis of allergic airway diseases. As a consequence, restoring barrier function might be a useful strategy in allergic airway disease treatment. This review, therefore, focuses on mechanisms that can restore epithelial barrier function.

\section{Barrier dysfunction in chronic inflammatory condi- tions: state-of-the-art}

For decades, chronic inflammatory diseases such as allergic rhinitis and allergic asthma were assumed to be caused by an unbalanced, over-reactive immunological response, mostly driven 
Table 1. Barrier dysfunction and pathology.

\begin{tabular}{|c|c|c|c|}
\hline Disease & Junctional proteins & Cells & Reference \\
\hline \multicolumn{4}{|l|}{ Upper and lower airways } \\
\hline Chronic rhinosinusitis & OCCL $\downarrow ; C L D N-4 \downarrow$ & HNEC, nasal biopsies & (44) \\
\hline Allergic rhinitis & OCCL $\downarrow ; Z O-1 \downarrow$ & HNEC, nasal biopsies & (26) \\
\hline Asthma & ZO- $1 \downarrow ; \beta$-catenin $\downarrow$ & Bronchial biopsies & (40) \\
\hline Asthma & OCCL $\downarrow ; Z O-1 \downarrow$ & HBEC, bronchial biopsies & (41) \\
\hline \multicolumn{4}{|l|}{ Skin } \\
\hline Atopic dermatitis & CLDN-1 $\downarrow ;$ CLDN-23 $\downarrow$ & Skin biopsies & (24) \\
\hline \multicolumn{4}{|l|}{ Gastrointestinal tract } \\
\hline Crohn's disease & OCCL $\downarrow ;$ CLDN-5 $\downarrow ;$ CLDN-8 $\downarrow ; C L D N-2 \downarrow$ & Colon biopsies & (36) \\
\hline Ulcerative colitis & -- & Colon biopsies & $(34,37)$ \\
\hline Functional dyspepsia & OCCL $\downarrow ; \beta$-catenin $\downarrow ;$ DSC2 $\downarrow ;$ DSG2 $\downarrow$ & Duodenal biopsies & (39) \\
\hline
\end{tabular}

by activated Th2 cells. However, this assumption cannot explain the full spectrum of the different inflammatory pathologies. Recently, there is growing interest in the epithelial barrier function as a major contributor to the pathophysiology of chronic inflammatory diseases. Opening of TJs facilitates the entrance of allergens and other harmful particles in the mucosal and submucosal region with the activation of the immune and inflammatory system as a consequence. On the other hand, opening of TJs also facilitates drainage of inflammation, which underscores the importance of a tight regulation of the epithelial barrier. We here give an overview of barrier dysfunction associated with allergic diseases in the skin ${ }^{(24)}$, the intestine ${ }^{(25)}$ and the airways (26) (Table 1).

Atopic dermatitis is a common chronic inflammatory condition of the skin ${ }^{(27)}$, with a high prevalence in the Westernized countries ${ }^{(28,29)}$. The skin maintains its integrity by two distinct barrier functions: the stratum corneum and the TJs ${ }^{(30)}$. Defects in the stratum corneum either by reduced lipid levels ${ }^{(31,32)}$ or by acquired/genetic mutation in the filaggrin gene ${ }^{(33)}$ or in other epidermal differentiation proteins, lead to the skin lesions typical for atopic dermatitis. TJ defects in the epidermis have recently been described by De Benedetto and colleagues ${ }^{(24)}$. Ex vivo studies on epidermal biopsies demonstrated a reduced expression of claudin-1 and claudin-23 with bioelectric defects (24). Interestingly, claudin-1 expression correlated inversely with the number of eosinophils in the skin, showing that claudin-1 affects the the immune response to environmental allergens. Increased epithelial permeability is also found in the intestine of patients with inflammatory bowel diseases like Crohn's disease or ulcerative colitis ${ }^{(34,35)}$. Early evidence obtained from freeze-fracture electron microscopy analysis revealed a decreased expression of tight junction strands in Crohn's disease together with the breakdown of TJ strands ${ }^{(35)}$. A clear reduction was found for TJ proteins occludin and the sealing claudins; claudin-5 and claudin-8. In contrast, an upregulation of claudin-2 protein was reported in patients with Crohn's disease ${ }^{(36)}$, resulting in a changed TJ architecture. In ulcerative colitis, the epithelial barrier is impaired by erosion/ulcer-type lesions and epithelial apoptosis causing local leaks and generalized TJ alterations increasing the basal permeability ${ }^{(34,37)}$. Functional dyspepsia is a gastro-intestinal disorder, affecting up to $20 \%$ of the population ${ }^{(38)}$. Despite the high prevalence, no clear treatment is available and the mechanisms contributing to this pathology are only partially revealed. Low grade inflammation in these subjects was found to be linked to increased intestinal permeability. Expression of occludin, E-cadherin and $\beta$-catenin were found to be decreased in intestinal biopsies ${ }^{(39)}$.

Evidence for barrier dysfunctions underlying the pathology in upper and lower airways has only recently been obtained. The first evidence for barrier dysfunction in the lower airways was found in 2008. Patients with mild asthma had a disturbed expression of ZO- 1 and $\beta$-catenin as shown by semi-quantitative immunofluorescence staining of bronchial biopsies ${ }^{(40)}$. The functional significance of the loss of ZO-1 and $\beta$-catenin was further illustrated in primary airway epithelial cell cultures and on bronchial biopsies. Primary bronchial epithelial cells, derived from asthmatics and cultured in vitro at air-liquid interface, showed a reduced epithelial integrity, together with a reduced expression of ZO-1 and occludin. This was also confirmed on bronchial biopsies of patients with asthma ${ }^{(41)}$. More detailed information on the regulation of epithelial barrier function in the lower airways has been extensively described elsewhere $(7,15,42,43)$.

In the upper airways, Soyka et al. ${ }^{(44)}$ have demonstrated a leaky epithelium in patients with chronic rhinosinusitis with/without nasal polyps. This leaky epithelium was linked with a decreased expression of occludin and claudin- 4 in nasal biopsies ${ }^{(44)}$. Our 


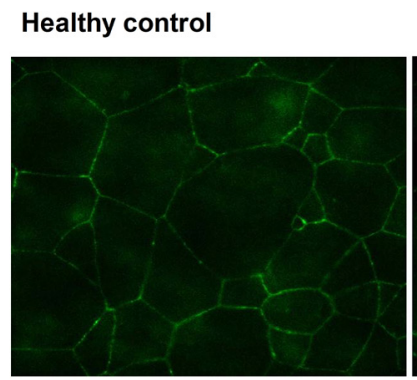

AR patient

Figure 2. Defective nasal epithelial barrier function in allergic rhinitis patients. Representative immunofluorescence staining for zonula occludens- 1 in nasal epithelial cell cultures from a healthy control and a patient with allergic rhinitis (AR). Green is zonula occludens-1. White arrows indicate defective epithelial integrity in AR patients.

group recently confirmed a disrupted TJ arrangement in the nasal cavity of patients with allergic rhinitis (Figure 2). We have shown by experiments in vitro, ex vivo and in vivo (in a mouse model of house dust mite induced allergic airway inflammation) that the barrier function is impaired and that this is due to a decreased expression of occludin and ZO- $1^{(26)}$. Interestingly, we showed that different TJ proteins (i.e. occludin and ZO-1) were involved in the pathology of allergic rhinitis compared to the TJ proteins (occludin and claudin-4) linked with the pathology of chronic rhinosinusitis, illustrating that the regulation of $\mathrm{TJ}$ function can be linked with the disease origin. Indeed, Kast et al. showed that each epithelial cell, depending on the site of isolation, had its own, unique expression pattern reflecting its specific function within the organism ${ }^{(45)}$. In the upper airways, Th2 inflammation is presumably the causative driving factor for epithelial dysfunction. Stimulation of epithelial cells from both allergic rhinitis and chronic rhinosinusitis patients with IL-4 resulted in a decreased epithelial integrity.

Although epithelial barrier defects are linked with different diseases; it remains unclear whether barrier dysfunction is a primary defect, participating to the onset of inflammation or only a consequence of sustained inflammation. Sunaert and colleagues showed that $\mathrm{t}$-cell induced colonic inflammation can change colonic epithelial integrity even before the development of colitis ${ }^{(46)}$. This suggests that defective epithelium is not only a consequence of inflammation but also an early defect that might actually strengthen inflammation by increasing the amount of foreign antigens in the mucosa. Noteworthy, epithelial cells from patients cultured in vitro for several weeks still show a decreased epithelial integrity, even in the absence of any inflammation. This point out towards an intrinsic effect in TJ function or a certain memory function in epithelial cells exposed to inflammation. Indeed, epigenetics might play a role in the memory effect. It has been shown that IL-13 induces long-lasting DNA methylation changes in airway epithelial cells from asthmatics ${ }^{(47)}$, contributing to the phenotype of asthma. In conclusion, defective epithelial barrier is linked with pathology but if this is a primary intrinsic effect, or a result of inflammation needs to be further elucidated.

\section{Models to study nasal barrier (dys)function}

Epithelial cell culture system in vitro

Primary nasal epithelial cells are the most relevant cells to be studied in vitro. A number of non-invasive techniques (nasal

Table 2. Models to study airway epithelial barrier (dys)function.

\begin{tabular}{|c|c|c|c|}
\hline Technique & Method of isolation & Advantages & Disadvantages \\
\hline \multicolumn{4}{|l|}{ Epithelial cell culture in vitro } \\
\hline \multirow[t]{2}{*}{ Primary epithelial cells } & Non-surgical; scraping & $\begin{array}{l}\text {-Well tolerated } \\
\text { - Repeated isolation possible } \\
\text { - No anesthesia }\end{array}$ & $\begin{array}{l}\text { - Limited cell numbers } \\
\text { - Culture difficulties }\end{array}$ \\
\hline & Surgical & $\begin{array}{l}\text { - Number of cells isolated (800.000 } \\
\text { cells/isolation) } \\
\text { - Comparison pathology and health }\end{array}$ & $\begin{array}{l}\text { - Regular access to tissue } \\
\text { - Inter variability } \\
\text { - Intra variability } \\
\text { - Culture difficulties }\end{array}$ \\
\hline Epithelial cell lines & -- & $\begin{array}{l}\text { - Unlimited number of cells } \\
\text { - Easy maintenance }\end{array}$ & $\begin{array}{l}\text { - Morphological differences } \\
\text { - Different cellular responses } \\
\text { - Biochemical characteristics are different }\end{array}$ \\
\hline \multicolumn{4}{|l|}{ Mucosal explant model } \\
\hline & Biopsies (local anaesthesia) & $\begin{array}{l}\text { - documents patients mucosal } \\
\text { status at time of isolation }\end{array}$ & $\begin{array}{l}\text { - Limited viability } \\
\text { - Limited number of biopsies harvested }\end{array}$ \\
\hline \multicolumn{4}{|l|}{ Murine models } \\
\hline & -- & $\begin{array}{l}\text { - Time- and dose-response experi- } \\
\text { ments } \\
\text { - Study of underlying inflammatory } \\
\text { mechanisms }\end{array}$ & $\begin{array}{l}\text { - Relevance needs to be confirmed in } \\
\text { humans }\end{array}$ \\
\hline
\end{tabular}


brushing, nasal scrapings, nasal smears, blown secretions) can be used to sample human nasal tissue or cells ${ }^{(48)}$. These nonsurgical procedures are well tolerated, do not require anesthesia and can easily be repeated. The main disadvantage of these techniques is the limited number of cells that can be recovered, and which is insufficient to grow a monolayer of epithelial cells. A higher number of nasal epithelial cells can be harvested from patients undergoing surgery. The harvested tissue (i.e. inferior turbinate) is enzymatically dissociated and so a purified epithelial cell population is obtained ${ }^{(26,49)}$. Although primary nasal epithelial cells are the best cells to study barrier function as they mimic the patient's condition, some limitations have to be considered. The number of epithelial cells isolated from one patient is still limited (average 800.000 cells/isolation), there is an interand intra-variability within cultures, a possible risk for bacterial contamination and technical difficulties in culturing ${ }^{(48)}$. Epithelial cell lines, derived from carcinomas, can be used to overcome the limitations of isolating primary epithelial cells from tissue. Different cells lines, from both the upper and lower airways, are available. The RPMI 2650 is a nasal epithelial cell line, derived from a human nasal anaplastic cell carcinoma, unfortunately with very poor differentiation ${ }^{(50)}$. In culture, RPMI 2650 cells do not express functional TJs and will not form a monolayer ${ }^{(51)}$. Therefore, they are not suitable to study epithelial barrier regulation. A better alternative is the human lung adenocarcinoma cell line, Calu-3. Calu-3 cells express TJs and form a polarized monolayer in vitro ${ }^{(52)}$. Another bronchial epithelial cell line is $16 \mathrm{HBE}{ }^{(53)}$, with similar culture characteristics as Calu-3 cells. Despite the easy maintenance and the unlimited amount of cells, epithelial cell lines do not have the same cellular responses, morphology and biochemical characteristics as primary cells. To evaluate epithelial barrier function, epithelial cells (either primary or epithelial cell lines) are seeded on transwell inserts. At a high seeding density, epithelial cells will grow to a confluent monolayer expressing TJs. After reaching a confluent monolayer, the liquid is removed from the apical side, so that epithelial cells can further differentiate at the air-liquid interface. By measuring the trans-epithelial electrical resistance (TEER), the epithelial integrity can be evaluated and followed over time. Besides TEER,
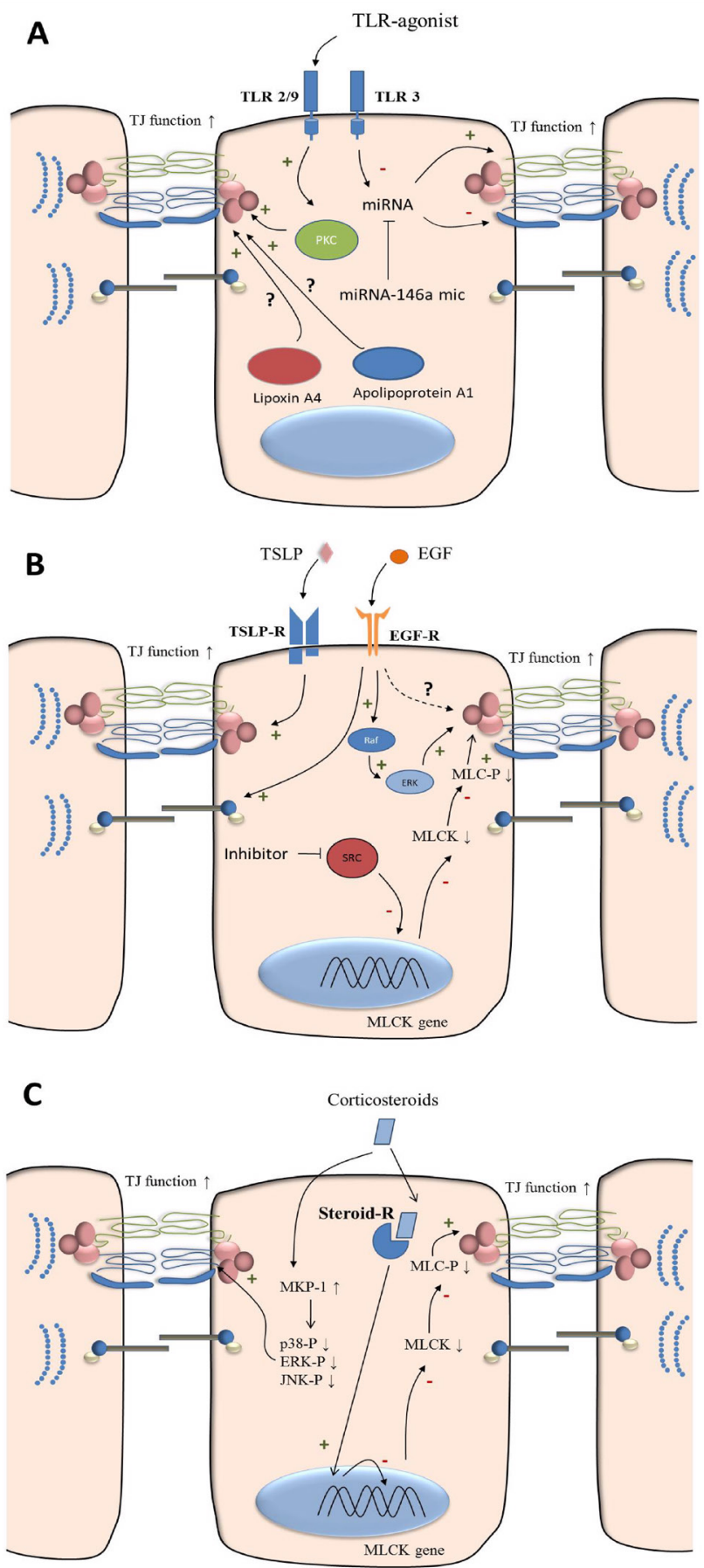

Figure 3. Restoration of epithelial barrier function. A). Activation of TLR2 or TLR9 stimulates PKC function and the expression of different TJ components. TLR3 activation results in expression of miRNAs with downregulation of TJ function. miRNAs function is suppressed by specific inhibitors, which results in upregulation of TJs and barrier function. Lipoxin A4 and apolipoprotein A1 can directly or indirectly (via mechanisms not fully understood) stimulate the expression of TJs. B). Binding of TSLP on its receptor stimulates the expression of TJ components. Activation of EGF-R results either in direct stimulation of TJ function or via its downstream components. Inhibition of SRC inhibits the activation of MLCK genes and therefore no phosphorylation of MLC and no internalization of TJs follows. C). Corticosteroids have different mechanisms of action. On the one hand, corticosteroids can bind to the steroid receptor and translocate to the nucleus where they suppress the activation of MLCK. Phosphorylation of MLC by MLCK is inhibited and the contraction of the actomyosin ring is prevented with no internalization of TJ complexes. On the other hand, corticosteroids can upregulate MKP-1 function. MKP-1 dephosphorylate p38, ERK, c-Jun N-terminal kinases resulting in an upregulation of barrier function. 
Table 3. Epithelial barrier restoring factors.

\begin{tabular}{|c|c|c|c|c|}
\hline Treatment & Tight junction proteins & Cells & Barrier function & Reference \\
\hline \multicolumn{5}{|l|}{ Innate immune function } \\
\hline Pam3CysSK4; Peptidoglycan & OCCL $\downarrow ; \mathrm{ZO}-1 \uparrow ; \mathrm{CLDN}-1 \uparrow$ & Calu-3 & TEER $\uparrow$; Fluorescein sodium $\downarrow$ & (59) \\
\hline Polyl:C & JAM-A $\downarrow$ & HNEC & TEER $=$ & (61) \\
\hline miRNA-146a & OCCL $\uparrow ; C L D N-1 \uparrow, J A M-A \uparrow$ & HNEC & TEER $\uparrow$ & (62) \\
\hline CpG-DNA & OCCL $\uparrow ; Z O-1,-2,-3 \uparrow ; C L D N-1,-4,-7 \uparrow$ & HBEC & TEER $\uparrow ;$ FD4 $\downarrow$ & (69) \\
\hline Apolipoprotein A1 & OCCL $\uparrow ; Z O-1 \uparrow$ & HBEC & TEER $\uparrow$ & (74) \\
\hline Lipoxin A4 & OCCL $\downarrow ; Z O-1 \uparrow ; C L D N-1 \uparrow$ & $16 \mathrm{HBE}$ & TEER $\uparrow$ & (76) \\
\hline \multicolumn{5}{|l|}{ Cytokines and growth factors } \\
\hline EGF & -- & $\begin{array}{l}\text { Caco-2; T-84 } \\
\text { cells }\end{array}$ & TEER $\uparrow$; Mannitol $\downarrow$ & (80) \\
\hline EGF & OCCL $\uparrow ;$ ZO-1 $\uparrow$ & Caco-2 & TEER $\uparrow$; Inulin $\downarrow$ & (81) \\
\hline EGF & OCCL $\uparrow ;$ ZO-1 $\uparrow$ & HBEC & TEER $\uparrow$ & (41) \\
\hline TSLP & OCCL $\uparrow ;$ CLDN-1,-4,-7 $\uparrow$ & HBEC & TEER $\uparrow$; Mannitol $\downarrow$ & (86) \\
\hline Src-kinase inhibitor & OCCL $\uparrow ;$ E-cadherin $\uparrow ; p 120$ catenin $\uparrow$ & HBEC & TEER $\uparrow ;$ FD4 $\downarrow$ & (89) \\
\hline \multicolumn{5}{|l|}{ Corticosteroids } \\
\hline Prednisone & -- & Patients & Lactulose/mannitol $\downarrow$ & (95) \\
\hline Dexamethasone & CLDN-4 $\uparrow$, CLDN-2 $\downarrow$ & Caco-2 & TEER $\uparrow ; \mathrm{LY}=$ & (96) \\
\hline Prednisone; Dexamethasone & -- & Caco-2 & TEER $\uparrow$; Inulin $\downarrow$ & (97) \\
\hline $\begin{array}{l}\text { Dexamethasone ; Fluticasone } \\
\text { propionate }\end{array}$ & OCCL $\uparrow ; Z O-1 \uparrow$ & Calu-3, 16HBE & TEER $\uparrow ;$ FD4 $\downarrow ;$ FD10 $\downarrow$ & (98) \\
\hline Fluticasone propionate & OCCL $\uparrow ; Z O-1 \uparrow$ & HNEC & TEER $\uparrow ;$ FD4 $\downarrow$ & (26) \\
\hline
\end{tabular}

CLDN: claudin; FD4: FITC-dextran 4kDa; FD10: FITC-dextran 10 kDa; HBEC: human bronchial epithelial cells; HNEC: human nasal epithelial cells; LY: luciferase yellow; OCCL: occludin; TEER: trans-epithelial electrical resistance; ZO: zonula occludens.

fluorescein-labeled dextran molecules can be used to study epithelial permeability as this tracer will migrate through the paracellular space from the apical side to the basolateral side.

\section{Explant model}

Although in vitro evaluation of epithelial barrier integrity and permeability is a widely used and accepted technique to study barrier function, it is a static and time consuming system. Nasal mucosal explants may be more suitable to evaluate mucosal integrity. Mucosal biopsies can easily be taken under local anesthetics and used as explants. With the use of Ussing chambers ${ }^{(54)}$, the mucosal integrity can be evaluated within hours post-isolation. A disadvantage of an explant model, however, is that stimulation studies that last for multiple hours cannot be performed on explants, as the viability of the explants is only preserved for a maximum of 6 hours.

\section{Murine models of allergic airway inflammation}

Murine models can be used to study the effect of allergens or other possible harmful substances on barrier function ${ }^{(55,56)}$. We have developed a mouse model to study changes in mucosal permeability induced by house dust mite extract ${ }^{(26)}$. Since house dust mite extract can cleave TJs ${ }^{(57)}$, the mucosal permeability can be evaluated using fluorescein labeled dextran. After intranasal instillation of the dextran tracer, the passage of the tracer to the serum can be evaluated and is a surrogate marker for mucosal permeability.

\section{Mechanisms involved in restoration of barrier dys- function}

As a defect in the epithelial barrier function is a hallmark of mucosal inflammation in different allergic diseases, restoration of barrier defects might reduce the excessive penetration of inhaled allergens and foreign particles into the submucosal regions, ultimately resolving the activation of the immune system and the occurrence of symptoms. A wide variety of possible mechanisms to restore barrier function are reported in literature (Table 3) (Figure 3).

\section{Innate immune function}

TLR are essential receptors in inducing an innate immune response against invading pathogens. TLR2 is recruited to the 
apical site of airway epithelial cells in the presence of pathogenic bacteria ${ }^{(58)}$. In Calu-3 bronchial epithelial cells, Pam3CysSK4 and peptidoglycan ligand-induced TLR2 activation significantly increased TEER in a dose-dependent manner, accompanied with a reduction in fluorescein sodium permeability ${ }^{(59)}$. This enhancement in barrier function was associated with an upregulation in expression levels of TJ proteins ZO-1 and claudin-1 and a downregulation of occludin.

TLR3 recognizes viral double-stranded RNA and its synthetic analogue polyinosinic-polycytidylic acid (polyl:C) ${ }^{(60)}$. Polyl:C induced activation of TLR3 in HNEC resulted in a decreased expression of JAM-A without changing epithelial integrity ${ }^{(61)}$. Interestingly, polyl:C activation resulted in the upregulation of micro RNA-146a via the TLR3-mediated signaling pathways phosphoinositide 3-kinase c-Jun N-terminal kinases and NF-KB (62). Micro-RNAs are a class of endogenous noncoding RNAs, regulating various biological processes ${ }^{(63)}$ including inflammatory responses in allergic inflammation in the nasal mucosa ${ }^{(64)}$ but also the formation of TJs and epithelial barriers ${ }^{(65,66)}$. When HNEC were treated with inhibitors of mircoRNAs, i.e. miR-146a mimic, this resulted in an increased expression of claudin-1, occludin and JAM-A together with an increased barrier function ${ }^{(62)}$. Furthermore, miR-146a mimic prevented the secretion of IL-8 and TNFa. Thus, interfering with microRNAs may represent a potential therapy for human upper airway viral diseases as Influenza A virus ${ }^{(67)}$ and respiratory syncytial virus ${ }^{(68)}$ induce the expression of microRNAs and downregulate barrier function. The activation of TLR9 by CpG-DNA or other TLR9 ligands enhanced barrier function of bronchial epithelial cells in vitro. Treatment with CpG-DNA resulted in the upregulation of ZO-1 and claudin-4, a sealing claudin ${ }^{(69)}$. However, treatment with CpG-DNA could not restore IL-13 induced barrier dysfunction. This means that inflammation inhibited or at least prevented the beneficial effect of CpG-DNA on restoration of epithelial barrier function in vitro. Nevertheless, a clinical study using TLR-9 agonist, BbG10, showed promising results for the control of asthma symptoms despite steroid withdrawal ${ }^{(70)}$.

Treatment with Apoliprotein A1 also enhanced barrier function. Apolipoprotein A1 is a high-density lipoprotein with antiinflammatory properties as it suppresses pro-inflammatory ERK signaling and NF-KB activation ${ }^{(71,72)}$. Apolipoprotein A1 is a key regulator of inflammation in murine models of asthma ${ }^{(73)}$ by promoting recovery of epithelial TJs. In patients with mild-tomoderate persistent asthma, lower levels of lung apolipoprotein A1 were found compared to controls ${ }^{(74)}$. When primary bronchial epithelial cells from mild-to-moderate asthmatics were treated in vitro locally with apolipoprotein $A 1$, occludin and ZO-1 was restored almost to the same levels as found in controls ${ }^{(74)}$. These data suggest that apolipoprotein A1 is a promising therapeutic strategy to repair defective epithelium by promoting the expression of $\mathrm{TJS}^{(74)}$.
Lipoxins are biologically active eicosanoids, secreted at sites of inflammation where they have anti-inflammatory properties ${ }^{(75)}$. In vitro stimulation of $16 \mathrm{HBE}$ bronchial epithelial cells with lipoxin A4 resulted in a time-dependent increase in TEER with an increase in ZO-1, occludin and claudin-1 expression ${ }^{(76)}$. This barrier promoting effect is mediated via the activation of the protein kinase $C$ signaling pathway, which stimulated the translocation of ZO-1 from the cytosol to the membrane (Figure 2A) ${ }^{(77) \text {. }}$

Effect of growth factors and cytokines on barrier regulation Growth factors such as epidermal growth factor (EGF) and keratinocyte growth factor have a potential positive effect on epithelial barrier integrity ${ }^{(41,78)}$. EGF plays a role in maintaining epithelial barrier homeostasis and is involved in healing of damaged airway mucosa ${ }^{(79)}$. EGF prevented oxidative stress-induced barrier disruption and $\mathrm{H}_{2} \mathrm{O}_{2}$ induced permeability ${ }^{(80,81)}$ in Caco-2 intestinal epithelial cells. EGF prevented $\mathrm{H}_{2} \mathrm{O}_{2}$ dependent phosphorylation of occludin and promoted cell growth and cell differentiation. Unlike to intestinal epithelial cells, EGF treatment could not reverse cigarette extract-induced epithelial injury in vitro in primary bronchial epithelial cells ${ }^{(41)}$. In contrast, Transforming Growth Factor- $\beta$, produced by almost all cell types in the lung, could prevent cigarette smoke induced barrier disruption by restoring ZO-1 and ZO-2 levels ${ }^{(82)}$.

Besides promoting cell growth, EGF also affects the epithelial barrier homeostasis. Treatment of in vitro cultured bronchial epithelial cells from asthmatic patients with EGF resulted in increased baseline barrier function, illustrated by increased TEER and TJ expression of occludin and ZO-1 ${ }^{(41)}$. Interestingly, only apical and not basolateral administration of EGF resulted in upregulation of TJ proteins in bronchial epithelial cells. The apical route of administration may therefore be a possible approach to restore barrier function but formulation, dose and method of administration need to be investigated further to limit the possible side-effects linked with EGF (41).

The type 2 cytokines IL-4 and IL-13 are capable of increasing epithelial permeability in $16 \mathrm{HBE}$ bronchial epithelial cells ${ }^{(83)}$. The role of these and other cytokines for barrier (dys)function has been reviewed by Georas et al. ${ }^{(7)}$ Epithelial-derived thymic stromal lymphopoietin (TSLP) is considered to be important in the pathogenesis of allergic asthma and allergic rhinitis ${ }^{(84,85)}$. TSLP is expressed primarily by epithelial cells in response to danger signals. Increased expression of TSLP has been documented in the nasal epithelium of allergic rhinitis patients ${ }^{\left({ }^{(86)}\right.}$. Although elevated levels of TSLP are linked with allergic diseases, treatment of human nasal epithelial cells with TSLP in vitro rapidly enhanced barrier function with upregulation of occludin, claudin-1, -4 and $-7{ }^{(86)}$. This suggests that TSLP preserves mucosal barrier function in the early stage of allergic rhinitis by promoting the closure of the nasal epithelial barrier, and as a result the penetration of 
allergens into the submucosal regions is limited.

Regardless of the correlation between TSLP and allergic rhinitis, high TNF levels are found in allergic airway diseases ${ }^{(87,88)}$. TNF has been shown to promote epithelial barrier dysfunction by decreasing the expression of occludin, claudins, E-cadherin and p120 catenin ${ }^{\left({ }^{89}\right)}$. TNF promotes release of TSLP, IL-6, IL-8, IL-1 $\beta$ and endogenous TNF. As TNF activates c-src kinases, tyrosine residues on occludin get phosphorylated, which results in the internalization of occludin with opening of the epithelial barrier ${ }^{(90)}$. SU6656, a c-src kinases antagonist ${ }^{(91)}$ reduced the effect of TNF on epithelial barrier permeability by inhibiting the loss of occludin, E-cadherin and p120 catenin This illustrates that inhibiting kinases can restore barrier function in bronchial epithelial cells of healthy donors in vitro. Interestingly, when bronchial epithelial cells from severe asthmatics, which have a significant lower TEER compared to those form healthy donors, were exposed in culture to RU6656, there was a significant decrease in macromolecular permeability with an upregulation of occludin, E-cadherin and p120 catenin. RU6656 treatment enhanced the barrier function in patients with asthma, suggesting that topical airway targeting of kinases might become a therapeutic approach to restore barrier function.

\section{Corticosteroids}

The first-line treatment of allergic diseases in both upper and lower airways is local application of corticosteroids ${ }^{\left({ }^{92}\right)}$. The therapeutic effect is mainly attributed to their anti-inflammatory properties resulting in a reduction of the infiltration of inflammatory cells into the affected tissue ${ }^{(93,94)}$. In patients with Crohn's disease, prednisolone treatment resulted in decreased gut permeability in the majority of patients, as reflected by the lactulose/mannitol ratio. This effect is commonly interpreted as a result of the resolution of inflammation ${ }^{\left({ }^{95}\right)}$. Various studies have however provided evidence that glucocorticoids can promote TJ sealing in the absence of inflammation. Treatment of Caco-2 intestinal epithelial cells with dexamethasone stimulated intestinal barrier function in a time- and dose-dependent manner with an upregulation of claudin- 4 and a downregulation of claudin-2 ${ }^{(96)}$. This effect was receptor-dependent and mediated by an increase in MAPK phosphatase-1 expression and activity. Nevertheless, the barrier restoring effect of dexamethasone on TJ expression in Caco-2 intestinal epithelial cells was not confirmed by Boivin et al. ${ }^{(97)}$. They did not find an upregulation of barrier function by dexamethasone or prednisolone. More interestingly, dexamethasone and prednisolone had a protective effect when Caco-2 cells were pre-exposed to TNF. This effect could be explained by corticosteroid induced suppression of the TNF induced increase in myosin light chain kinase gene activity, protein expression, and subsequent opening of the intestinal TJ barrier ${ }^{(97)}$.

Compared to the intestine, little is known about the effect of corticosteroids in both upper and lower airways. Treatment of 2 bronchial epithelial cell lines, i.e. Calu-3 and 16HBE, with dexamethasone and fluticasone propionate resulted in an increased epithelial barrier integrity by stimulating the expression of occludin and ZO-1 ${ }^{(98)}$. Our group recently showed that fluticasone propionate enhanced barrier integrity in primary nasal epithelial cells from both healthy controls and house dust mite allergic rhinitis patients ${ }^{(26)}$. Moreover, allergic rhinitis patients using steroids on a daily basis had a significantly stronger barrier function compared to patients not using steroids.

The positive effect of corticosteroids on TJs is orchestrated via different molecular mechanisms (Figure 2C). Corticosteroids stimulate the MAPK phosphatase-1 pathway, which regulates the anti-inflammatory effects of corticosteroids by dephosphorylating ERK and p38 ${ }^{(99)}$. Besides MAPK phosphatase-1, myosin light chain kinases also play a central role in the regulation of epithelial TJ permeability ${ }^{(100,101)}$. Activated myosin light chain kinases phosphorylate myosin light chain and stimulate the contraction of the peri-junctional actomyosin ring. As a result, TJs are internalized and therefore the TJ opens ${ }^{(100)}$. Corticosteroid treatment inhibits myosin light chain kinase and thus inhibits the internalization of TJs. In summary, different treatment strategies can result in the restoration of impaired epithelial barrier function, providing new insights and new therapeutic targets for allergic airway diseases.

\section{Unmet needs to nasal barrier dysfunction}

Allergic rhinitis, which is defined as a symptomatic inflammation of the nasal mucosa, affects up to $30 \%$ of the total population ${ }^{(92,102)}$. Despite the different treatment modalities like intranasal steroids, anti-histamines, leukotriene receptor antagonists and immunotherapy ${ }^{(103,104)}$, there is still a fraction of patients that is uncontrolled with adequate therapy ${ }^{(105,106)}$. Identifying these difficult-to-treat patients is of crucial importance, as nasal epithelial barrier defects may play a pivotal role in uncontrolled disease. Nonetheless, compared to the gastrointestinal tract, no clinical test is available to evaluate nasal epithelial barrier function in vitro. In the gastrointestinal tract, the lactulose/mannitol ratio can be used to measure small intestinal permeability ${ }^{(107)}$, providing information about the degree of epithelial damage It would therefore be of great interest to be able to investigate in a clinical setting barrier dysfunction in patients with allergic rhinitis. This will help us better understand the pathology of allergic rhinitis. Moreover, using a clinical diagnostic test, the success of different treatment options can be evaluated which will help us understand why more than $20 \%$ of patients are uncontrolled.

\section{Conclusion}

Epithelial barrier defects are linked with pathology in the airways, gut and skin. Whether barrier defects represent a primary 
genetic defect or a secondary phenomenon resulting from inflammatory mechanisms needs to be investigated further. Restoring barrier function or preventing barrier damage may represent a new treatment target. The development of molecules that restore or at least up-regulate epithelial barrier integrity might be very useful for treatment of allergic diseases, especially in difficult-to-treat patients who fail to respond to conventional therapy.

\section{Authorship contribution}

BS performed literature review and drafted the manuscript. SFS and JLC performed a critical review of the manuscript and sig- nificantly contributed to the manuscript draft and editing. GB, CAA and PHW performed a critical review of the manuscript.

\section{Disclosures}

The authors are supported by grants from the Belgian Federal Government (IUAP P7/30), the institute for Science and technology Flanders (IWT) (TBM project 130260) and the research council of the KU Leuven (GOA 2009/07 and 14/011). P.W.H. is a recipient of a senior researcher fellowship from the Fund of Scientific Research (FWO), Flanders, Belgium. S.F.S. supported by the research council of KU Leuven (PDMK/14/189).

\section{References}

1. Koch S, Nusrat A. The life and death of epithelia during inflammation: lessons learned from the gut. Annu Rev Pathol 2012; 7:3560.

2. Bals R, Hiemstra PS. Innate immunity in the lung: how epithelial cells fight against respiratory pathogens. Eur Respir J 2004; 23:327-33.

3. Parker D, Prince A. Innate immunity in the respiratory epithelium. Am J Respir Cell Mol Biol 2011; 45:189-201.

4. Lambrecht BN, Hammad H. The airway epithelium in asthma. Nat Med 2012; 18:68492.

5. Sha Q, Truong-Tran AQ, Plitt JR, Beck LA, Schleimer RP. Activation of airway epithelial cells by toll-like receptor agonists. Am J Respir Cell Mol Biol 2004; 31:358-64.

6. Vroling $A B$, Fokkens WJ, van Drunen $C M$ How epithelial cells detect danger: aid ing the immune response. Allergy 2008 63:1110-23

7. Georas SN, Rezaee F. Epithelial barrier function: at the front line of asthma immunology and allergic airway inflammation. J Allergy Clin Immunol 2014; 134:509-20.

8. Shen L, Weber CR, Raleigh DR, Yu D, Turner JR. Tight junction pore and leak pathways: a dynamic duo. Annu Rev Physiol 2011; 73:283-309.

9. Nelson WJ, Nusse R. Convergence of Wht beta-catenin, and cadherin pathways. Science 2004; 303:1483-7.

10. Hartsock A, Nelson WJ. Adherens and tight junctions: structure, function and connections to the actin cytoskeleton. Biochim Biophys Acta 2008; 1778:660-9.

11. Brooke MA, Nitoiu D, Kelsell DP. Cell-cell connectivity: desmosomes and disease. Pathol 2012; 226:158-71.

12. Niessen CM. Tight junctions/adherens junctions: basic structure and function. J Invest Dermatol 2007; 127:2525-32.

13. Johnson JL, Najor NA, Green KJ. Desmosomes: regulators of cellular signaling and adhesion in epidermal health and disease. Cold Spring Harb Perspect Med 2014; 4:a015297.

14. Schulzke JD, Fromm M. Tight junctions: molecular structure meets function. Ann N Y Acad Sci 2009; 1165:1-6.

15. Rezaee F, Georas SN. Breaking barriers. New insights into airway epithelial barrier function in health and disease. Am J Respir Cell Mol Biol 2014; 50:857-69.

16. Gunzel D, Yu AS. Claudins and the modulation of tight junction permeability. Physiol Rev 2013; 93:525-69.

17. Van Itallie CM, Anderson JM. Claudins and epithelial paracellular transport. Annu Rev Physiol 2006; 68:403-29.

18. Amasheh S, Meiri N, Gitter AH, Schoneberg T, Mankertz J, Schulzke JD, et al. Claudin-2 expression induces cation-selective channels in tight junctions of epithelial cells. J Cell Sci 2002; 115:4969-76.

19. Alexandre MD, Lu Q, Chen YH. Overexpression of claudin-7 decreases the paracellular $\mathrm{Cl}$ - conductance and increases the paracellular $\mathrm{Na}+$ conductance in LLCPK1 cells. J Cell Sci 2005; 118:2683-93.

20. Inai T, Kobayashi J, Shibata Y. Claudin-1 contributes to the epithelial barrier function in MDCK cells. Eur J Cell Biol 1999; 78:849-55.

21. Hou J, Renigunta A, Yang J, Waldegger S. Claudin-4 forms paracellular chloride channel in the kidney and requires claudin-8 for tight junction localization. Proc Natl Acad Sci U S A 2010; 107:18010-5.

22. Utech M, Bruwer M, Nusrat A. Tight junctions and cell-cell interactions. Methods Mol Biol 2006; 341:185-95.

23. Mehta S, Nijhuis A, Kumagai T, Lindsay J, Silver A. Defects in the adherens junction complex (E-cadherin/ beta-catenin) in inflammatory bowel disease. Cell Tissue Res 2015; 360:749-60.

24. De Benedetto A, Rafaels NM, McGirt LY, Ivanov Al, Georas SN, Cheadle C, et al. Tight junction defects in patients with atopic dermatitis. J Allergy Clin Immunol 2011; 127:773-86 e1-7.

25. Schulzke JD, Ploeger S, Amasheh M, Fromm A, Zeissig S, Troeger $\mathrm{H}$, et al. Epithelial tight junctions in intestinal inflammation. Ann N Y Acad Sci 2009; 1165:294-300.

26. Steelant B, Farre R, Wawrzyniak P, Belmans J, Dekimpe E, Vanheel $H$, et al. Impaired barrier function in patients with house dust mite-induced allergic rhinitis is accompanied by decreased occludin and zonula occludens-1 expression. J Allergy Clin Immunol. 2016;137(4):1043-1053.

27. Leung DY, Boguniewicz M, Howell MD, Nomura I, Hamid QA. New insights into atopic dermatitis. J Clin Invest 2004; 113:651-7.

28. Vickery BP. Skin barrier function in atopic dermatitis. Curr Opin Pediatr 2007; 19:89-93.

29. Spergel JM, Paller AS. Atopic dermatitis and the atopic march. J Allergy Clin Immunol 2003; 112:S118-27.

30. Madison KC. Barrier function of the skin: "la raison d'etre" of the epidermis. J Invest Dermatol 2003; 121:231-41.

31. Imokawa G. Lipid abnormalities in atopic dermatitis. J Am Acad Dermatol 2001; 45:S29-32.

32. Pilgram GS, Vissers DC, van der Meulen H, Pavel S, Lavrijsen SP, Bouwstra JA, et al. Aberrant lipid organization in stratum corneum of patients with atopic dermatitis and lamellar ichthyosis. J Invest Dermatol 2001; 117:710-7

33. Palmer CN, Irvine AD, Terron-Kwiatkowski A, Zhao Y, Liao H, Lee SP, et al. Common lossof-function variants of the epidermal barrier protein filaggrin are a major predisposing factor for atopic dermatitis. Nat Genet 2006; 38:441-6.

34. Schmitz H, Barmeyer C, Fromm M, Runkel N, Foss HD, Bentzel CJ, et al. Altered tight junction structure contributes to the impaired epithelial barrier function in ulcerative colitis. Gastroenterology 1999; 116:301-9.

35. Marin ML, Greenstein AJ, Geller SA, Gordon $R E$, Aufses $A H, J$ r. A freeze fracture study of Crohn's disease of the terminal ileum: changes in epithelial tight junction organization. Am J Gastroenterol 1983; 78:537-47.

36. Zeissig S, Burgel N, Gunzel D, Richter J, Mankertz J, Wahnschaffe $U$, et al. Changes in expression and distribution of claudin 2, 5 and 8 lead to discontinuous tight junctions and barrier dysfunction in active Crohn's disease. Gut 2007; 56:61-72.

37. Gitter AH, Wullstein F, Fromm M, Schulzke JD. Epithelial barrier defects in ulcerative colitis: characterization and quanti- 
fication by electrophysiological imaging Gastroenterology 2001; 121:1320-8.

38. Tack J, Bisschops R, Sarnelli G. Pathophysiology and treatment of functional dyspepsia. Gastroenterology 2004; 127:1239-55

39. Vanheel $H$, Vicario M, Vanuytsel $T$, Van Oudenhove L, Martinez C, Keita AV, et al. Impaired duodenal mucosal integrity and low-grade inflammation in functional dyspepsia. Gut 2014; 63:262-71.

40. de Boer WI, Sharma HS, Baelemans SM, Hoogsteden HC, Lambrecht BN, Braunstahl GJ. Altered expression of epithelial junctional proteins in atopic asthma: possible role in inflammation. Can J Physiol Pharmacol 2008; 86:105-12.

41. Xiao C, Puddicombe SM, Field S, Haywood J, Broughton-Head V, Puxeddu I, et al. Defective epithelial barrier function in asthma. J Allergy Clin Immunol 2011; 128:54956 e $1-12$.

42. Losifidis T, Garratt LW, Coombe DR, Knight DA, Stick SM, Kicic A. Airway epithelial repair in health and disease: Orchestrator or simply a player? Respirology. 2016;21(3):438-48.

43. Davies DE. Epithelial barrier function and immunity in asthma. Ann Am Thorac Soc 2014; 11 Suppl 5:S244-51.

44. Soyka MB, Wawrzyniak P, Eiwegger T, Holzmann D, Treis A, Wanke K, et al. Defective epithelial barrier in chronic rhinosinusitis: the regulation of tight junctions by IFN-gamma and IL-4. J Allergy Clin Immunol 2012; 130:1087-96 e10.

45. Kast JI, Wanke K, Soyka MB, Wawrzyniak P, Akdis D, Kingo K, et al. The broad spectrum of interepithelial junctions in skin and lung. J Allergy Clin Immunol 2012; 130:544-7 e4.

46. Suenaert $P$, Maerten $P$, Van Assche $G$, Van Driessche W, Geboes K, Bulteel V, et al. Effects of T cell-induced colonic inflammation on epithelial barrier function. Inflamm Bowel Dis 2010; 16:1322-31.

47. Nicodemus-Johnson J, Naughton KA, Sudi J, Hogarth K, Naurekas ET, Nicolae DL, et al. Genome-Wide Methylation Study Identifies an IL-13-induced Epigenetic Signature in Asthmatic Airways. Am J Respir Crit Care Med 2016; 193:376-85.

48. Dimova S, Brewster ME, Noppe M, Jorissen $M$, Augustijns P. The use of human nasal in vitro cell systems during drug discovery and development. Toxicol In Vitro 2005; 19:107-22.

49. Bobic S, van Drunen CM, Callebaut I, Hox V, Jorissen M, Fokkens WJ, et al. Dexamethasone-induced apoptosis of freshly isolated human nasal epithelial cells concomitant with abrogation of IL-8 production. Rhinology 2010; 48:401-7.

50. Merkle HP, Ditzinger G, Lang SR, Peter $H$, Schmidt MC. In vitro cell models to study nasal mucosal permeability and metabolism. Adv Drug Deliv Rev 1998; 29:51-79.

51. De Fraissinette A, Brun R, Felix $H$, Vonderscher J, Rummelt A. Evaluation of the human cell line RPMI 2650 as an in vitro nasal model. Rhinology 1995; 33:194-8.

52. Shen $B Q$, Finkbeiner WE, Wine JJ, Mrsny RJ, Widdicombe JH. Calu-3: a human airway epithelial cell line that shows CAMPdependent $\mathrm{Cl}$ - secretion. Am J Physiol 1994; 266:L493-501.

53. Cozens AL, Yezzi MJ, Kunzelmann K, Ohrui T, Chin L, Eng K, et al. CFTR expression and chloride secretion in polarized immorta human bronchial epithelial cells. Am Respir Cell Mol Biol 1994; 10:38-47.

54. Wallon C, Braaf $Y$, Wolving M, Olaison G, Soderholm JD. Endoscopic biopsies in Ussing chambers evaluated for studies of macromolecular permeability in the human colon. Scand J Gastroenterol 2005; 40:58695.

55. Hox V, Vanoirbeek JA, Callebaut I, Bobic S, De Vooght V, Ceuppens J, et al. Airway exposure to hypochlorite prior to ovalbumin induces airway hyperreactivity without evidence for allergic sensitization. Toxicol Lett 2011; 204:101-7.

56. Hox V, Vanoirbeek JA, Alpizar YA, Voedisch S, Callebaut I, Bobic S, et al. Crucial role of transient receptor potential ankyrin 1 and mast cells in induction of nonallergic airway hyperreactivity in mice. Am J Respir Crit Care Med 2013; 187:486-93.

57. Wan H, Winton HL, Soeller C, Tovey ER, Gruenert DC, Thompson PJ, et al. Der p 1 facilitates transepithelial allergen delivery by disruption of tight junctions. J Clin Invest 1999; 104:123-33.

58. Soong G, Reddy B, Sokol S, Adamo R, Prince A. TLR2 is mobilized into an apical lipid raft receptor complex to signal infection in airway epithelial cells. J Clin Invest 2004; 113:1482-9

59. Ragupathy S, Esmaeili F, Paschoud S, Sublet E, Citi S, Borchard G. Toll-like receptor 2 regulates the barrier function of human bronchial epithelial monolayers through atypical protein kinase $\mathrm{C}$ zeta, and an increase in expression of claudin-1. Tissue Barriers 2014; 2:e29166

60. Alexopoulou L, Holt AC, Medzhitov R, Flavell RA. Recognition of double-stranded RNA and activation of NF-kappaB by Toll-like receptor 3. Nature 2001; 413:732-8.

61. Ohkuni T, Kojima T, Ogasawara N, Masaki T, Fuchimoto J, Kamekura R, et al. Poly(l:C) reduces expression of JAM-A and induces secretion of IL-8 and TNF-alpha via distinct NF-kappaB pathways in human nasal epithelial cells. Toxicol Appl Pharmacol 2011; 250:29-38.

62. Miyata R, Kakuki T, Nomura K, Ohkuni T, Ogasawara N, Takano K, et al. Poly(l:C) induced microRNA-146a regulates epithelial barrier and secretion of proinflammatory cytokines in human nasal epithelial cells. Eur J Pharmacol 2015; 761:375-82

63. Farh KK, Grimson A, Jan C, Lewis BP, Johnston WK, Lim LP, et al. The widespread impact of mammalian MicroRNAs on mRNA repression and evolution. Science 2005; 310:1817-21.
64. Suojalehto H, Toskala E, Kilpelainen M, Majuri ML, Mitts C, Lindstrom I, et al. MicroRNA profiles in nasal mucosa of patients with allergic and nonallergic rhinitis and asthma. Int Forum Allergy Rhinol 2013; 3:612-20.

65. Ye D, Guo S, Al-Sadi R, Ma TY. MicroRNA regulation of intestinal epithelial tight junction permeability. Gastroenterology 2011; 141:1323-33.

66. Cichon C, Sabharwal H, Ruter C, Schmidt MA. MicroRNAs regulate tight junction proteins and modulate epithelial/endothelial barrier functions. Tissue Barriers 2014; 2:e944446.

67. Buggele WA, Johnson KE, Horvath CM. Influenza A virus infection of human respiratory cells induces primary microRNA expression. J Biol Chem 2012; 287:31027-40.

68. Kojima T, Go M, Takano K, Kurose M, Ohkuni T, Koizumi J, et al. Regulation of tight junctions in upper airway epithelium. Biomed Res Int 2013; 2013:947072.

69. Kubo T, Wawrzyniak P, Morita H, Sugita K, Wanke K, Kast Jl, et al. CpG-DNA enhances the tight junction integrity of the bronchial epithelial cell barrier. J Allergy Clin Immunol 2015.

70. Beeh KM, Kanniess F, Wagner F, Schilder C, Naudts I, Hammann-Haenni A, et al. The novel TLR-9 agonist QbG10 shows clinical efficacy in persistent allergic asthma. J Allergy Clin Immunol 2013; 131:866-74.

71. Robbesyn F, Garcia V, Auge N, Vieira O, Frisach MF, Salvayre $R$, et al. HDL counterbalance the proinflammatory effect of oxidized LDL by inhibiting intracellular reactive oxygen species rise, proteasome activation, and subsequent NF-kappaB activation in smooth muscle cells. FASEB J 2003; 17:7435 .

72. Navab M, Anantharamaiah GM, Fogelman AM. The role of high-density lipoprotein in inflammation. Trends Cardiovasc Med 2005; 15:158-61.

73. Yao X, Dai C, Fredriksson K, Dagur PK, McCoy JP, Qu X, et al. 5A, an apolipoprotein $\mathrm{A}-\mathrm{I}$ mimetic peptide, attenuates the induction of house dust mite-induced asthma. J Immunol 2011; 186:576-83.

74. Park SW, Lee EH, Lee EJ, Kim HJ, Bae DJ, Han S, et al. Apolipoprotein A1 potentiates lipoxin A4 synthesis and recovery of allergen-induced disrupted tight junctions in the airway epithelium. Clin Exp Allergy 2013; 43:914-27.

75. Bonnans C, Chanez P, Chavis C. Lipoxins in asthma: potential therapeutic mediators on bronchial inflammation? Allergy 2004; 59:1027-41.

76. Grumbach Y, Quynh NV, Chiron R, Urbach $V$. LXA4 stimulates ZO-1 expression and transepithelial electrical resistance in human airway epithelial (16HBE140-) cells. Am J Physiol Lung Cell Mol Physiol 2009; 296:L101-8.

77. Chen ML, Pothoulakis C, LaMont JT. Protein kinase $\mathrm{C}$ signaling regulates $\mathrm{ZO}-1$ transloca- 
tion and increased paracellular flux of T84 colonocytes exposed to Clostridium difficile toxin A. J Biol Chem 2002; 277:4247-54.

78. Tillie-Leblond I, Gosset P, Le Berre R, Janin A, Prangere T, Tonnel AB, et al. Keratinocyte growth factor improves alterations of lung permeability and bronchial epithelium in allergic rats. Eur Respir J 2007; 30:31-9.

79. Carpenter G, Cohen S. Epidermal growth factor. J Biol Chem 1990; 265:7709-12.

80. Rao R, Baker RD, Baker SS. Inhibition of oxidant-induced barrier disruption and protein tyrosine phosphorylation in Caco-2 cell monolayers by epidermal growth factor. Biochem Pharmacol 1999; 57:685-95.

81. Basuroy S, Seth A, Elias B, Naren AP, Rao R. MAPK interacts with occludin and mediates EGF-induced prevention of tight junction disruption by hydrogen peroxide. Biochem J 2006; 393:69-77.

82. Schamberger AC, Mise N, Jia J, Genoyer E, Yildirim AO, Meiners S, et al. Cigarette smoke-induced disruption of bronchial epithelial tight junctions is prevented by transforming growth factor-beta. Am J Respir Cell Mol Biol 2014; 50:1040-52.

83. Saatian B, Rezaee F, Desando S, Emo J, Chapman T, Knowlden S, et al. Interleukin-4 and interleukin-13 cause barrier dysfunction in human airway epithelial cells. Tissue Barriers 2013; 1:e24333.

84. Holgate ST. Epithelium dysfunction in asthma. J Allergy Clin Immunol 2007; 120:123344; quiz 45-6.

85. Ying S, O'Connor B, Ratoff J, Meng Q, Mallett $K$, Cousins D, et al. Thymic stromal lymphopoietin expression is increased in asthmatic airways and correlates with expression of Th2-attracting chemokines and disease severity. J Immunol 2005; 174:8183-90.

86. Kamekura R, Kojima T, Koizumi J, Ogasawara N, Kurose M, Go M, et al. Thymic stromal lymphopoietin enhances tight-junction barrier function of human nasal epithelial cells. Cell Tissue Res 2009; 338:283-93.

87. Brightling C, Berry M, Amrani Y. Targeting TNF-alpha: a novel therapeutic approach for asthma. J Allergy Clin Immunol 2008; 121:510; quiz 1-2.

88. Babu SK, Puddicombe SM, Arshad HH Wilson SJ, Ward J, Gozzard N, et al. Tumor necrosis factor alpha (TNF-alpha) autoregulates its expression and induces adhesion molecule expression in asthma. Clin Immunol 2011; 140:18-25.

89. Hardyman MA, Wilkinson E, Martin E Jayasekera NP, Blume C, Swindle EJ, et al.
TNF-alpha-mediated bronchial barrier disruption and regulation by src-family kinase activation. J Allergy Clin Immunol 2013; 132:665-75 e8.

90. Dorfel MJ, Huber O. Modulation of tight junction structure and function by kinases and phosphatases targeting occludin. J Biomed Biotechnol 2012; 2012:807356.

91. Huang S, Dudez T, Scerri I, Thomas MA, Giepmans BN, Suter S, et al. Defective activation of c-Src in cystic fibrosis airway epithelial cells results in loss of tumor necrosis factor-alpha-induced gap junction regulation. J Biol Chem 2003; 278:8326-32.

92. Greiner AN, Hellings PW, Rotiroti G, Scadding GK. Allergic rhinitis. Lancet 2011; 378:2112-22.

93. Schleimer RP. Effects of glucocorticosteroids on inflammatory cells relevant to their therapeutic applications in asthma. Am Rev Respir Dis 1990; 141:S59-69.

94. Schwiebert LM, Beck LA, Stellato C, Bickel CA, Bochner BS, Schleimer RP. Glucocorticosteroid inhibition of cytokine production: relevance to antiallergic actions. J Allergy Clin Immunol 1996; 97:143-52.

95. Wild GE, Waschke KA, Bitton A, Thomson AB The mechanisms of prednisone inhibition of inflammation in Crohn's disease involve changes in intestinal permeability, mucosal TNFalpha production and nuclear factor kappa B expression. Aliment Pharmacol Ther 2003; 18:309-17.

96. Fischer A, Gluth M, Weege F, Pape UF, Wiedenmann B, Baumgart DC, et al. Glucocorticoids regulate barrier function and claudin expression in intestinal epithelial cells via MKP-1. Am J Physiol Gastrointest Liver Physiol 2014; 306:G218-28.

97. Boivin MA, Ye D, Kennedy JC, Al-Sadi R, Shepela C, Ma TY. Mechanism of glucocorticoid regulation of the intestinal tight junction barrier. Am J Physiol Gastrointest Liver Physiol 2007; 292:G590-8.

98. Sekiyama A, Gon Y, Terakado M, Takeshita I, Kozu Y, Maruoka S, et al. Glucocorticoids enhance airway epithelial barrier integrity. Int Immunopharmacol 2012; 12:350-7.

99. Wancket LM, Frazier WJ, Liu Y. Mitogenactivated protein kinase phosphatase (MKP)-1 in immunology, physiology, and disease. Life Sci 2012; 90:237-48.

100. Ma TY, Boivin MA, Ye D, Pedram A, Said HM. Mechanism of TNF-\{alpha\} modulation of Caco-2 intestinal epithelial tight junction barrier: role of myosin light-chain kinase protein expression. Am J Physiol Gastrointest Liver Physiol 2005; 288:G42230.

101.Ma TY, Iwamoto GK, Hoa NT, Akotia V, Pedram A, Boivin MA, et al. TNF-alphainduced increase in intestinal epithelial tight junction permeability requires NF-kappa B activation. Am J Physiol Gastrointest Liver Physiol 2004; 286:G36776.

102. Hox V, Steelant B, Fokkens W, Nemery B, Hellings PW. Occupational upper airway disease: how work affects the nose. Allergy 2014; 69:282-91.

103. Cornelis M, Rombaux $P$, Jorissen M, Hellings PW. Nationwide survey on immunotherapy practice by ENT specialists. Rhinology 2014; 52:72-7.

104. Petalas K, Durham SR. Allergen immunotherapy for allergic rhinitis. Rhinology 2013; 51:99-110.

105. Bousquet PJ, Bachert C, Canonica GW, Casale TB, Mullol J, Klossek JM, et al. Uncontrolled allergic rhinitis during treatment and its impact on quality of life: a cluster randomized trial. J Allergy Clin Immunol 2010; 126:666-8 e 1-5.

106. Hellings PW, Fokkens WJ, Akdis C, Bachert C, Cingi C, Dietz de Loos D, et al. Uncontrolled allergic rhinitis and chronic rhinosinusitis: where do we stand today? Allergy 2013; 68:1-7.

107. Odenwald MA, Turner JR. Intestinal permeability defects: is it time to treat? Clin Gastroenterol Hepatol 2013; 11:1075-83.

Prof. Dr. Peter W. Hellings

Clinical Division of Otorhinolaryngo-

logy, Head and Neck Surgery

University Hospitals Leuven

KU Leuven

Kapucijnenvoer 33

3000 Leuven

Belgium

Tel.: +32-16-33 2338

Fax: +32-16-34 6035

E-mail:

Peter.hellings@med.kuleuven.be 